PROCEEDINGS OF THE

AMERICAN MATHEMATICAL SOCIETY

Volume 124, Number 11, November 1996

\title{
ELEMENTARY OPERATORS AND INVARIANT SUBALGEBRAS
}

\author{
KEITH J. COATES
}

(Communicated by Palle E. T. Jorgensen)

\begin{abstract}
We provide an example of an elementary operator which leaves invariant a nest algebra but which cannot be written as a finite sum of multiplications each of which leaves the nest algebra invariant. We also prove that the given operator lies in the completely bounded norm closure of the linear span of the multiplications which leave the nest algebra invariant.
\end{abstract}

Let $\mathcal{A}$ be a Banach algebra and let $A, B \in \mathcal{A}$. A multiplication (operator) $A \cdot B$ : $\mathcal{A} \rightarrow \mathcal{A}$ is defined by the equation $(A \cdot B)(X)=A X B, X \in \mathcal{A}$. An elementary operator $R$ is an operator of the form $R=\sum_{i=1}^{n} A_{i} \cdot B_{i}$, where $n$ is finite. Clearly, $R$ can have different representations $\sum C_{t} \cdot D_{t}$. The length $l$ of $R$ is defined to be the smallest number of multiplication terms required for any representation of $R$.

Elementary operators have been studied in great detail in recent years. Both their spectral theory and their structural theory have received much attention (cf. $[\mathrm{AF}, \mathrm{Cu}, \mathrm{F}])$. The range inclusion problem for elementary operators $[\mathrm{F}]$ can be stated as follows: If $\mathcal{I}$ is an ideal in $\mathcal{A}$, and if $\operatorname{Ran}(R) \subseteq \mathcal{I}$, must $R$ be expressible as $\sum_{i=1}^{n} A_{i} \cdot B_{i}$ with $\operatorname{Ran}\left(A_{i} \cdot B_{i}\right) \subseteq \mathcal{I}, i=1,2, \ldots, n$ ? Positive results have been obtained with certain hypotheses $[\overline{\mathrm{AF}}]$.

Let $\mathcal{C}$ be a subalgebra of $\mathcal{A}$. David Larson has suggested the study of $\mathcal{E}_{\mathcal{C}}(\mathcal{A})$, the algebra of elementary operators on $\mathcal{A}$ which leave $\mathcal{C}$ invariant. With $\mathcal{A}=B\left(l_{2}\right)$ and $\mathcal{C}=\mathcal{T}_{\infty}$, the nest algebra of upper triangular operators with respect to the standard basis in $l_{2}$, we provide in Theorem 3 an example of an $R \in \mathcal{E}_{\mathcal{C}}(\mathcal{A})$ which has no representation $\sum_{t=1}^{m} C_{t} \cdot D_{t}$ such that $C_{t} \cdot D_{t} \in \mathcal{E}_{\mathcal{C}}(\mathcal{A}), t=1,2, \ldots, m$. (This example was discussed, without proof, in [Co].) We also demonstrate in Proposition 4 that $R$ lies in the completely bounded norm closed linear span of the length one elements of $\mathcal{E}_{\mathcal{C}}(\mathcal{A})$

$\mathcal{T}_{\infty}$ is a commutative subspace lattice (CSL) algebra. Recall that a CSL algebra is a reflexive algebra whose lattice of invariant subspaces is commutative. For finite dimensional CSL algebras there is no operator like the operator $R$ of the previous paragraph.

Proposition 1. Let $\mathcal{C}$ be a CSL subalgebra of $\mathcal{M}_{n}$, and let $R \in \mathcal{E}_{\mathcal{C}}\left(\mathcal{M}_{n}\right)(R \neq$ $0)$. Then there exists a representation $R=\sum_{t=1}^{m} C_{t} \cdot D_{t}$ such that $C_{t} \cdot D_{t} \in$ $\mathcal{E}_{\mathcal{C}}\left(\mathcal{M}_{n}\right), 1 \leq t \leq m$.

Proof. Let $R=\sum_{s=1}^{p} A_{s} \cdot B_{s}$. Since $\mathcal{C}$ is a CSL algebra, it admits a star diagram, that is, there exists a subset $I \times J \subseteq\{1,2, \ldots, n\} \times\{1,2, \ldots, n\}$ such that $\mathcal{C}=$ $\left\{\left(a_{i j}\right): a_{i j} \in C,(i, j) \in I \times J\right\}[\mathrm{KL}]$.

Received by the editors September 8, 1994 and, in revised form, May 18, 1995.

1991 Mathematics Subject Classification. Primary 47D25; Secondary 47A15. 
Let $A_{1}=\left(a_{i j}\right)=\sum a_{i j} E_{i j}, B_{1}=\left(b_{i j}\right)=\sum b_{i j} E_{i j}$. With this, we see that we can expand $A_{1} \cdot B_{1}$ as a linear combination of multiplications involving only pairs of matrix units: $A_{1} \cdot B_{1}=\sum_{i, j, k, l} \alpha_{i j k l}\left(E_{i j} \cdot E_{k l}\right)$. Expanding $A_{2} \cdot B_{2}, \ldots, A_{p} \cdot B_{p}$ in like manner and combining like terms, we arrive at

$$
R=\sum \beta_{i j k l}\left(E_{i j} \cdot E_{k l}\right)
$$

Let $E_{a b} \in \mathcal{C}$ and assume that $R\left(E_{a b}\right) \neq 0$. Then

$$
R\left(E_{a b}\right)=\sum_{i, j} \beta_{i a b j}\left(E_{i a} \cdot E_{b j}\right) E_{a b}=\sum_{i, j} \beta_{i a b j} E_{i j}
$$

and there is a $(c, d)$ such that $\beta_{c a b d} \neq 0$. Then $E_{c d} \in \mathcal{C}$, for if not, we would have that $\sum \beta_{i a b j} E_{i j} \notin \mathcal{C}$, contradicting our hypothesis. So every nonzero multiplication $\beta_{i a b j}\left(E_{i a} \cdot E_{b j}\right)$ maps $E_{a b}$ into $\mathcal{C}$ and every multiplication in (1) either annihilates $E_{a b}$ or maps it onto a nonzero element of $\mathcal{C}$. Thus $\sum \beta_{i j k l}\left(E_{i j} \cdot E_{k l}\right)$ is an expansion of $R$ such that each multiplication lies in $\mathcal{E}_{\mathcal{C}}\left(\mathcal{M}_{n}\right)$.

Note: The conclusion of Proposition 1 can be false if $\mathcal{C}$ is not a CSL subalgebra of $\mathcal{M}_{n}$ (see Example 5).

Clearly, the number of terms in (1) can be arbitrarily large for arbitrarily large $n$. The observation of this fact in a specific instance will facilitate the proof of Theorem 3.

Lemma 2. Let $n>2$, and let $\mathcal{T}_{n} \subseteq \mathcal{M}_{n}$ be the algebra of upper triangular $n \times n$ matrices. There exists an $R \in \mathcal{E}_{\mathcal{T}_{n}}\left(\mathcal{M}_{n}\right)$ of length two such that for any representation $R=\sum_{t=1}^{m} C_{t} \cdot D_{t}$ with each $C_{t} \cdot D_{t} \in \mathcal{E}_{\mathcal{T}_{n}}\left(\mathcal{M}_{n}\right)$, we must have $m \geq \log _{2} n$.

Proof. Let $\left\{\alpha_{1}, \alpha_{2}, \ldots, \alpha_{n-1}\right\}$ be a sequence of distinct complex numbers and define the following four matrices in $\mathcal{M}_{n}$ :

$$
\begin{gathered}
A_{1}=\sum_{i=2}^{n} E_{i i}, \quad B_{1}=\sum_{j=2}^{n} \alpha_{j-1} E_{j(j-1)}, \\
A_{2}=\sum_{k=2}^{n} \alpha_{k-1} E_{k k}, \quad B_{2}=\sum_{l=2}^{n}-E_{l(l-1)} .
\end{gathered}
$$

Consider the elementary operator $R=A_{1} \cdot B_{1}+A_{2} \cdot B_{2}$. By Proposition $1, R$ expands as

$$
\sum_{i=2}^{n} \sum_{j=2}^{n}\left(\alpha_{j-1}-\alpha_{i-1}\right)\left(E_{i i} \cdot E_{j(j-1)}\right)
$$

$R \in \mathcal{E}_{\mathcal{T}_{n}}\left(\mathcal{M}_{n}\right)$ since it takes strictly upper triangular matrix units to upper triangular matrix units, and the diagonal matrix units are annihilated.

Since the $\alpha_{k}$ 's are all distinct, any expansion of $R$ must include nonzero multiples of every one of the terms

$$
E_{i i} \cdot E_{j(j-1)}
$$

for $i \neq j, 2 \leq i, j \leq n$. On the other hand, if $C \cdot D \in \mathcal{E}_{\mathcal{T}_{n}}\left(\mathcal{M}_{n}\right)$ is to contain a nonzero multiple of $E_{i i} \cdot E_{j(j-1)}$ then $C$ must contain a nonzero multiple of $E_{i i}$ and $D$ must contain a nonzero multiple of $E_{j(j-1)}$. Thus, if $C \cdot D$ contains nonzero multiples of $E_{i i} \cdot E_{j(j-1)}$ and $E_{k k} \cdot E_{l(l-1)}$ then it must also contain a nonzero multiple of $E_{k k} \cdot E_{j(j-1)}$. If $C \cdot D$ is to leave $\mathcal{T}_{n}$ invariant, we must have $k \neq j$. 
Now suppose that $R=\sum_{t=1}^{m} C_{t} \cdot D_{t}$. For each $i$ let $W_{i}$ be the set of $t$ 's for which $C_{t} \cdot D_{t}$ contains a nonzero multiple of $E_{i i} \cdot E_{k(k-1)}$. We claim the sets $W_{i}$ are all distinct. If $i \neq j$, the last paragraph implies that there is a $t_{0} \in W_{i}$ such that $C_{t_{0}} \cdot D_{t_{0}}$ contains a nonzero multiple of $E_{i i} \cdot E_{j(j-1)}$. But, also by the last paragraph, this means that $C_{t_{0}} \cdot D_{t_{0}}$ cannot contain nonzero multiples of any operator of the form $E_{j j} \cdot E_{l(l-1)}$. This tells us that $t_{0} \notin W_{j}$, so $W_{j} \neq W_{i}$.

Thus, the total number of sets $W_{i}$ (that is, $n-1$ ) is no greater than the total number of distinct nonempty subsets of $\{1,2, \ldots, m\}$, which is $2^{m}-1$. The result follows.

Theorem 3. There exists an elementary operator $R \in \mathcal{E}_{\mathcal{T}_{\infty}}\left(B\left(l_{2}\right)\right)$ of length two which cannot be written as a finite sum $\sum_{t=1}^{m} C_{t} \cdot D_{t}$ of multiplications with $C_{t} \cdot D_{t} \in$ $\mathcal{E}_{\mathcal{T}_{\infty}}\left(B\left(l_{2}\right)\right)$ for $1 \leq t \leq m$.

Proof. Let $\alpha=\left(\alpha_{1}, \alpha_{2}, \ldots\right)$ be a sequence in $l_{\infty}$ with the $\alpha_{i}$ 's all distinct. Form the following four operators in $B\left(l_{2}\right)$ :

$$
\begin{aligned}
& A_{1}=\sum_{i=2}^{\infty} E_{i i}, \quad B_{1}=\sum_{j=2}^{\infty} \alpha_{j-1} E_{j(j-1)}, \\
& A_{2}=\sum_{k=2}^{\infty} \alpha_{k-1} E_{k k}, \quad B_{2}=\sum_{l=2}^{\infty}-E_{l(l-1)} .
\end{aligned}
$$

Let $R=A_{1} \cdot B_{1}+A_{2} \cdot B_{2}$. Again, routine computations show that $R \in \mathcal{E}_{\mathcal{T}_{\infty}}\left(B\left(l_{2}\right)\right)$, but $A_{1} \cdot B_{1} \notin \mathcal{E}_{\mathcal{T}_{\infty}}\left(B\left(l_{2}\right)\right), A_{2} \cdot B_{2} \notin \mathcal{E}_{\mathcal{T}_{\infty}}\left(B\left(l_{2}\right)\right)$.

Suppose we can write $R$ as a finite sum $\sum_{t=1}^{m} C_{t} \cdot D_{t}$ with $C_{t} \cdot D_{t} \in \mathcal{E}_{\mathcal{T}_{\infty}}\left(B\left(l_{2}\right)\right)$, $1 \leq t \leq m$. With $n$ fixed and $P_{n}$ the projection onto the first $n$ basis vectors, let $X^{\prime}=P_{n} X P_{n} \in \mathcal{M}_{n}$ for $X \in B\left(l_{2}\right)$. Let $R^{\prime}=A_{1}^{\prime} \cdot B_{1}^{\prime}+A_{2}^{\prime} \cdot B_{2}^{\prime}$. Then $R^{\prime}$ is an elementary operator of the type constructed in Lemma 2. Further, $R^{\prime}=$ $\sum_{t=1}^{m} C_{t}^{\prime} \cdot D_{t}^{\prime}$ with $C_{t}^{\prime} \cdot D_{t}^{\prime} \in \mathcal{E}_{\mathcal{T}_{n}}\left(\mathcal{M}_{n}\right)$ for $1 \leq t \leq m$. Lemma 2 therefore implies that $m \geq \log _{2} n$. But this must be true for all $n>2$ with $m$ fixed, an impossibility.

We conclude that $R$ cannot be written as a finite sum $\sum_{t=1}^{m} C_{t} \cdot D_{t}$ with $C_{t} \cdot D_{t} \in$ $\mathcal{E}_{\mathcal{T}_{\infty}}\left(B\left(l_{2}\right)\right)$

If $\mathcal{A}$ is a $\mathrm{C}^{*}$-algebra and $\phi: \mathcal{A} \rightarrow \mathcal{A}$ is a bounded linear operator, define $\phi_{n}$ : $M_{n}(\mathcal{A}) \rightarrow M_{n}(\mathcal{A})$ by the equation $\phi_{n}\left(\left(a_{i j}\right)\right)=\left(\phi\left(a_{i j}\right)\right)_{i j}$. If $\sup _{n}\left\|\phi_{n}\right\|<\infty$, say that $\phi$ is completely bounded and define the completely bounded norm $\|\phi\|_{c b}$ of $\phi$ to be this supremum. $C B(\mathcal{A})$, the algebra of completely bounded operators, contains the elementary operators as a proper (nonclosed) subalgebra.

If $\mathcal{A} \odot \mathcal{B}$ is the algebraic tensor product of two operator algebras $\mathcal{A}, \mathcal{B}$, define $\|u\|_{h}$, the Haagerup norm of $u \in \mathcal{A} \odot \mathcal{B}$, by

$$
\|u\|_{h}=\inf \left\{\left\|\left(a_{1}, a_{2}, \ldots, a_{n}\right)\right\|\left\|\left(b_{1}, b_{2}, \ldots, b_{n}\right)^{t}\right\|: u=\sum_{i=1}^{n} a_{i} \otimes b_{i}\right\} .
$$

The closure of $\mathcal{A} \odot \mathcal{B}$ in the topology defined by this norm is known as the Haagerup tensor product of $\mathcal{A}$ and $\mathcal{B}$ and is denoted by $\mathcal{A} \otimes_{h} \mathcal{B}$. For an excellent overview of the theory of completely bounded operators and the Haagerup tensor product, see [ChrSi]. 
There are rich connections between the structures of $C B(\mathcal{A})$ and of $\mathcal{A} \odot \mathcal{A}$. In $[\mathrm{CSi}]$ it is shown that the map $\theta: \mathcal{A} \odot \mathcal{A} \rightarrow C B(\mathcal{A})$, given by $\theta(a \otimes b)(x)=a x b, x \in$ $\mathcal{A}$, is an isometry.

Proposition 4. Let $\mathcal{F}$ be the linear span of the length one elements of $\mathcal{E}_{\mathcal{T}_{\infty}}\left(B\left(l_{2}\right)\right)$, and let $\overline{\mathcal{F}}$ be the closure of $\mathcal{F}$ in the completely bounded norm topology. Then $R \in \overline{\mathcal{F}}$, where $R$ is the operator constructed in Theorem 3.

Proof. Without loss of generality, assume $\|\alpha\|_{\infty} \leq 1$. Let $\epsilon$ be given, and let $\beta=$ $\left(\beta_{1}^{\prime}, \beta_{2}^{\prime}, \ldots\right) \in l_{\infty}$ be a simple (i.e., finitely valued) $\epsilon / 3$ approximation of $\alpha$ such that $\|\beta\|_{\infty} \leq 1$. Let $\left\{\beta_{1}, \beta_{2}, \ldots, \beta_{m}\right\}$ be the set of distinct values which $\beta$ assumes. Form the following four operators in $B\left(l_{2}\right)$ :

$$
\begin{gathered}
C_{1}=\sum_{i=2}^{\infty} E_{i i}, \quad D_{1}=\sum_{j=2}^{\infty} \beta_{j-1}^{\prime} E_{j(j-1)}, \\
C_{2}=\sum_{k=2}^{\infty} \beta_{k-1}^{\prime} E_{k k}, \quad D_{2}=\sum_{l=2}^{\infty}-E_{l(l-1)},
\end{gathered}
$$

and let $S=C_{1} \cdot D_{1}+C_{2} \cdot D_{2}$. We first show that $S \in \mathcal{F}$. Let $Q_{1}, Q_{2}, \ldots, Q_{m}$ be diagonal projections such that $\sum_{i=1}^{m} \beta_{i} Q_{i}=C_{2}$. Let $T_{1}, T_{2}, \ldots, T_{m}$ be operators with only ones and zeroes on the first subdiagonal and zeroes elsewhere such that $\sum_{i=1}^{m} \beta_{i} T_{i}=D_{1}$.

Now, for $E_{i j} \in \mathcal{T}_{\infty}, E_{i i}\left(E_{i j}\right) E_{j(j-1)}=E_{i(j-1)} \in \mathcal{T}_{\infty}$ as long as $i \neq j$ (for then $i<j$, so that $i \leq j-1)$. Thus, $Q_{r} \cdot T_{s} \in \mathcal{E}_{\mathcal{T}_{\infty}}\left(B\left(l_{2}\right)\right)$ for $r \neq s$. We have

$$
\begin{aligned}
C_{1} \cdot D_{1}+C_{2} \cdot D_{2}=\left(\left(\sum Q_{r}\right) \cdot\left(\sum \beta_{s} T_{s}\right)\right)-\left(\left(\sum \beta_{r} Q_{r}\right) \cdot\left(\sum T_{s}\right)\right) \\
=\sum_{1 \leq r, s \leq m} \beta_{s}\left(Q_{r} \cdot T_{s}\right)-\sum_{1 \leq r, s \leq m} \beta_{r}\left(Q_{r} \cdot T_{s}\right)=\sum_{r \neq s} \beta_{s}\left(Q_{r} \cdot T_{s}\right)-\beta_{r}\left(Q_{r} \cdot T_{s}\right) .
\end{aligned}
$$

Each term in the above (finite) sum lies in $\mathcal{E}_{\mathcal{T}_{\infty}}\left(B\left(l_{2}\right)\right)$, so that $S=C_{1} \cdot D_{1}+C_{2} \cdot D_{2} \in$ $\mathcal{F}$.

We now show that $\|S-R\|_{c b}<\epsilon$. We have that $\left\|A_{1}-C_{1}\right\|=\left\|B_{2}-D_{2}\right\|=0$, and $\left\|A_{2}-C_{2}\right\|<\epsilon / 3,\left\|B_{1}-D_{1}\right\|<\epsilon / 3$. Consider the following operator vectors:

$$
\left(0, \sqrt{\frac{\epsilon}{3}} C_{1}, \sqrt{\frac{3}{\epsilon}}\left(A_{2}-C_{2}\right), \sqrt{\frac{\epsilon}{3}} C_{2}\right), \quad\left(\sqrt{\frac{\epsilon}{3}} B_{1}, \sqrt{\frac{3}{\epsilon}}\left(B_{1}-D_{1}\right), \sqrt{\frac{\epsilon}{3}} B_{2}, 0\right)^{t} .
$$

By construction, each has norm less than $\sqrt{\epsilon}$. Thus, $\epsilon$ is larger than

$$
\begin{gathered}
\left\|0 \otimes \sqrt{\frac{\epsilon}{3}} B_{1}+\sqrt{\frac{\epsilon}{3}} C_{1} \otimes \sqrt{\frac{3}{\epsilon}}\left(B_{1}-D_{1}\right)+\sqrt{\frac{3}{\epsilon}}\left(A_{2}-C_{2}\right) \otimes \sqrt{\frac{\epsilon}{3}} B_{2}+\sqrt{\frac{\epsilon}{3}} C_{2} \otimes 0\right\|_{h} \\
=\left\|\left(A_{1}-C_{1}\right) \otimes B_{1}+C_{1} \otimes\left(B_{1}-D_{1}\right)+\left(A_{2}-C_{2}\right) \otimes B_{2}+C_{2} \otimes\left(B_{2}-D_{2}\right)\right\|_{h} \\
=\left\|\left[\left(A_{1} \otimes B_{1}\right)+\left(A_{2} \otimes B_{2}\right)\right]-\left[\left(C_{1} \otimes D_{1}\right)+\left(C_{2} \otimes D_{2}\right)\right]\right\|_{h} .
\end{gathered}
$$

Theorem 4.3 in [CSi] now implies that $\|S-R\|_{c b}<\epsilon$.

Since $\epsilon$ was arbitrary, $R \in \overline{\mathcal{F}}$. 
We close with a finite dimensional example, which demonstrates that if $\mathcal{C}$ is not a CSL subalgebra of $\mathcal{M}_{n}$, then the conclusion of proposition 1 can be false.

Example 5. Let $\mathcal{A}=\mathcal{M}_{2}$, the algebra of $2 \times 2$ matrices over $C$. Let $\mathcal{C}$ be the triangular Toeplitz subalgebra

$$
\mathcal{C}=\left\{\left(\begin{array}{cc}
\lambda & \mu \\
0 & \lambda
\end{array}\right): \lambda, \mu \in \mathbf{C}\right\}
$$

Let

$$
A_{1}=B_{1}=\left(\begin{array}{ll}
0 & 0 \\
0 & 1
\end{array}\right), \quad A_{2}=\left(\begin{array}{cc}
0 & 0 \\
1 & 0
\end{array}\right), \quad B_{2}=\left(\begin{array}{cc}
0 & -1 \\
0 & 0
\end{array}\right),
$$

and let $R=A_{1} \cdot B_{1}+A_{2} \cdot B_{2}$. It is not difficult to verify that

$$
R\left(\begin{array}{ll}
a & b \\
c & d
\end{array}\right)=\left(\begin{array}{cc}
0 & 0 \\
0 & d-a
\end{array}\right) \quad(\text { so } R \neq 0)
$$

and

$$
R\left(\begin{array}{cc}
\lambda & \mu \\
0 & \lambda
\end{array}\right)=\left(\begin{array}{cc}
0 & 0 \\
0 & \lambda-\lambda
\end{array}\right)=\left(\begin{array}{ll}
0 & 0 \\
0 & 0
\end{array}\right)
$$

Thus, $R \in \mathcal{E}_{\mathcal{C}}(\mathcal{A})$ (in fact, $R$ annihilates $\mathcal{C}$ ).

Routine computations now show that $R$ does not lie in (the norm closure of) the linear span of length one elements of $\mathcal{E}_{\mathcal{C}}(\mathcal{A})$.

\section{ACKNOWLEDGEMENT}

The author wishes to thank both David Larson and Roger Smith for helpful conversations during the writing of this paper.

\section{REFERENCES}

[AF] C. Apostol and L. A. Fialkow, Structural properties of elementary operators, Can. J. Math. 38 (1986), 1485-1524. MR 88c:47071

[CSi] A. Chatterjee and A. M. Sinclair, An isometry from the Haagerup tensor product into the completely bounded operators, J. Operator Theory 28 (1992), 65-78. MR 95j:46065

[ChrSi] E. Christensen and A. M. Sinclair, A survey of completely bounded operators, Bull. London Math. Soc. 21 (1989), 417-448. MR 91b:46051

[Co] K. Coates, Elementary operators and subalgebras, Algebraic Methods in Operator Theory, Birkhauser, Boston, 1994, pp. 49-51. MR 96b:47039

[Cu $\quad$ R. E. Curto, Spectral theory of elementary operators, Elementary Operators and Applications, World Scientific, 1992, pp. 3-52. MR 93i:47041

[F] L. A. Fialkow, The range inclusion problem for elementary operators, Michigan Math. J. 34 (1987), 451-459. MR 89a:47052

[KL] J. Kraus and D. Larson, Reflexivity and distance formulae, Proc. London Math. Soc. 53 (1986), 340-356. MR 87m:47100

Department of Mathematics, Illinois Wesleyan University, Bloomington, Illinois 61702

E-mail address: kcoates@sun.iwu.edu 